Citation: Khan, A., Prasad, R.P., "Adaptive Control for Synchronization of Identical and Non-Identical Chaotic Systems with Unknown Parameters". Journal of Engineering Technology and Applied Sciences 5 (2) 2020 : 77 92.

\title{
ADAPTIVE CONTROL FOR SYNCHRONIZATION OF IDENTICAL AND NON-IDENTICAL CHAOTIC SYSTEMS WITH UNKNOWN PARAMETERS
}

\author{
Ayub Khan ${ }^{a}$, Ram P. Prasad ${ }^{\text {* }}$ \\ ${ }^{a}$ Department of Mathematics, Faculty of Natural Science, Jamia Millia Islamia, Delhi, India \\ akhan12@jmi.ac.in \\ ${ }^{b}$ Deaperment of Mathematics Hansraj College, University of Delhi, Delhi, India \\ ram.mbhudu@gmail.com (*corresponding author)
}

\begin{abstract}
In this paper, adaptive control theory is utilized to derive nonlinear controllers for the synchronization of two identical and non-identical chaotic systems with unknown parameters. Based on the Lyapunov stability theory, the adaptive control laws for synchronization controllers associated with adaptive update laws of system parameters are developed to make the states of two identical and non-identical systems synchronized. The feasibility of the obtained results are validated with numerical simulation.
\end{abstract}

Keywords: Synchronization, adaptive control, Lyapunov stability

\section{Introduction}

Chaotic phenomena in numerous natural and social systems have attracted great interested since E. Lorenz discovered the first physical chaotic attractor [1]. Chaos is also an interesting phenomenon of nonlinear systems. A deterministic chaotic system has some remarkable dynamics characteristics [2], such as system evolution sensitive to the initial conditions and broad spectrum of Fourier transform. It can be treated as a carrier to modulate signals that have the random characteristics. It also has the overall stability. When we use a chaotic signal to drive two identical systems, the two systems or certain parts of them will have the synchronous behaviour, which does well for confidential communication.

Since synchronization of chaos has been put forward by Pecora and Carroll in 1990 [3], the phenomenon and its application in secure communications attracts much attention $[4,5,6,7,8$, $9,10]$. Until now, many types of synchronization of chaotic system have been proposed, such 
as generalized synchronization [11, 12], phase synchronization [13, 14], lag synchronization [15], anti-synchronization [16] and so on. At the same time, a wide variety of approaches for the synchronization and control of chaotic system have also been put forward in recent years $[17,18,19,20,21]$, such as PC method, adaptive control method [22], observer control method [23], fuzzy control method, global synchronization method, etc.

Recently, Hyperchaotic systems have attracted much attention in nonlinear area. Hyperchaotic system has more than one positive Lyapunov exponent which generates more complex dynamics than the low- dimensional chaotic system. Therefore Hyperchaotic system has much wider application than the low-dimensional chaotic system. For example, the adoption of Hyperchaotic system has been proposed for secure communication and the presence of more than one positive Lyapunov exponent clearly improves the security of the communication scheme [24].

In this paper, we study chaos synchronization of two identical Pan, and two identical Chen systems and non-identical Pan and Chen chaotic systems by adaptive control method. It is assumed that the parameters of the systems are unknown.

\section{Mathematical description}

Consider the drive chaotic system in the form of

$\dot{x}=f(x)+F(x) \alpha$

where $x \in \Omega_{1} \subset R^{n}$ is the state vector, $\alpha \in R^{m}$ is the unknown parameter vector of the system, $f(x)$ is an $n \times 1$ matrix, $F(x)$ is an $n \times m$ matrix, the elements $F_{i j}(x)$ in matrix $F(x)$ satisfies $F_{i j}(x) \in L_{\infty}$ for $x \in \Omega_{1} \subset R^{n}$. On the other hand, the response system is assumed by

$\dot{y}=g(y)+G(y) \beta+u$

where $y \in \Omega_{2} \subset R^{n}$ is the state vector, $\beta \in R^{q}$ is the unknown parameter vector of the system, $g(y)$ is an $n \times 1$ matrix, $G(y)$ is an $n \times q$ matrix, $u \in R^{n}$ in control input vector, the elements $G_{i j}(y)$ in matrix $G(y)$ satisfies $G_{i j}(y) \in L_{\infty}$ for $y \in \Omega_{2} \subset R^{n}$.

Let $e=y-x$ is the synchronization error vector. Our goal is to design controller $u$ such that the trajectory of the response system (2) with initial condition $y_{0}$ can asymptotically approaches the drive system (1) with initial condition $x_{0}$ and finally implement the synchronization, that is,

$\lim _{t \rightarrow \infty}\|e\|=\lim _{t \rightarrow \infty}\left\|y\left(t, y_{0}\right)-x\left(t, x_{0}\right)\right\|=0$

where $\|$.$\| is the Euclidean norm.$

\subsection{Adaptive synchronization controller design}

Theorem 2.1 If the nonlinear control is selected as 
$u=-f(x)-F(x) \hat{\alpha}-g(y)-G(y) \hat{\beta}-k e$,

And adaptive laws of parameters are taken as

$\dot{\hat{\alpha}}=[F(x)]^{T} e$,

$\dot{\hat{\beta}}=[G(y)]^{T} e$,

Then the response system (2) can synchronize the drive system (1), where $k>0$ is a constant, $\hat{\alpha}$ and $\hat{\beta}$ are respectively, estimations of the unknown parameters $\alpha$ and $\beta$ where $\alpha$ and $\beta$ are constants.

Proof. From eqns. (1) \& (2), we get the error dynamical system as follows

$\dot{e}=F(x)(\alpha-\hat{\alpha})+G(y)(\beta-\widehat{\beta})-k e$

Let $\tilde{\alpha}=\alpha-\hat{\alpha}, \tilde{\beta}=\beta-\hat{\beta}$. If the Lyapunov function is chosen as

$V(e, \tilde{\alpha}, \tilde{\beta})=\frac{1}{2}\left[e^{T} e+(\alpha-\widehat{\alpha})^{T}(\alpha-\widehat{\alpha})+(\beta-\widehat{\beta})^{T}(\beta-\widehat{\beta})\right]$

Then the derivative of $V$ along the trajectory of the error dynamical system is as follows

$$
\begin{aligned}
& \dot{V}(e, \tilde{\alpha}, \tilde{\beta})=\dot{e}^{T} e+(\alpha-\widehat{\alpha})^{T} \dot{\tilde{\alpha}}+(\beta-\widehat{\beta})^{T} \dot{\tilde{\beta}} \\
& =[F(x)(\alpha-\hat{\alpha})+G(y)(\beta-\widehat{\beta})-k e]^{T} e-(\alpha-\widehat{\alpha})^{T}[F(x)]^{T} e-(\beta-\widehat{\beta})^{T}[G(y)]^{T} e \\
& =-k e^{T}, e<0
\end{aligned}
$$

As long as $e \neq 0$, thus, $\frac{d V}{d t}<0$ for $V>0$, and the proof follows from the Theorem of Lyapunov stability.

Remark 2.2 Most typical chaotic systems can be described by (1), such as the Pan system, the Chen system.

Remark 2.3 If system (1) and system (2) satisfies $f()=.g($.$) and F()=.G($.$) . Then the structure$ of system (1) and system (2) are identical. Therefore, Theorem 2.1 is also applicable to the adaptive synchronization of two identical chaotic systems with unknown parameters.

The chaotic Pan system is given by

$\dot{x}_{1}=a\left(x_{2}-x_{1}\right)$

$\dot{x}_{2}=c x_{1}-x_{1} x_{3}$

$\dot{x}_{3}=x_{1} x_{2}-b x_{3}$

where $x_{1}, x_{2}$ and $x_{3}$ are state variables and $a, b$ and $c$ are constant. If $a=10, b=8 / 3$ and $c=16$ then the Pan system is chaotic. 


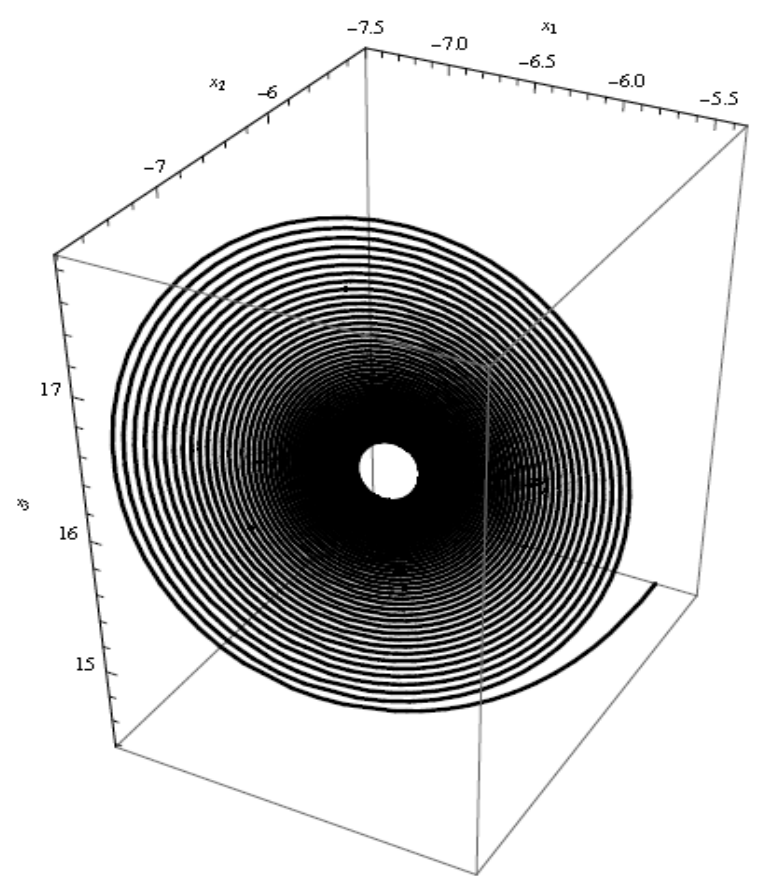

Figure 1. Chaotic attractor of Pan System when $a=10, b=8 / 3$ and $c=16$

Also Chen system is given by

$$
\begin{aligned}
& \dot{x}_{1}=\alpha\left(x_{2}-x_{1}\right) \\
& \dot{x}_{2}=\gamma x_{1}-\alpha x_{1}-x_{1} x_{3}+\gamma x_{2} \\
& \dot{x}_{3}=x_{1} x_{2}-\beta x_{3}
\end{aligned}
$$

where $x_{1}, x_{2}$ and $x_{3}$ are state variables and $\alpha, \beta$ and $\gamma$ are constant. If $\alpha=35, \beta=3$ and $\gamma=28$ then the Chen system is chaotic.

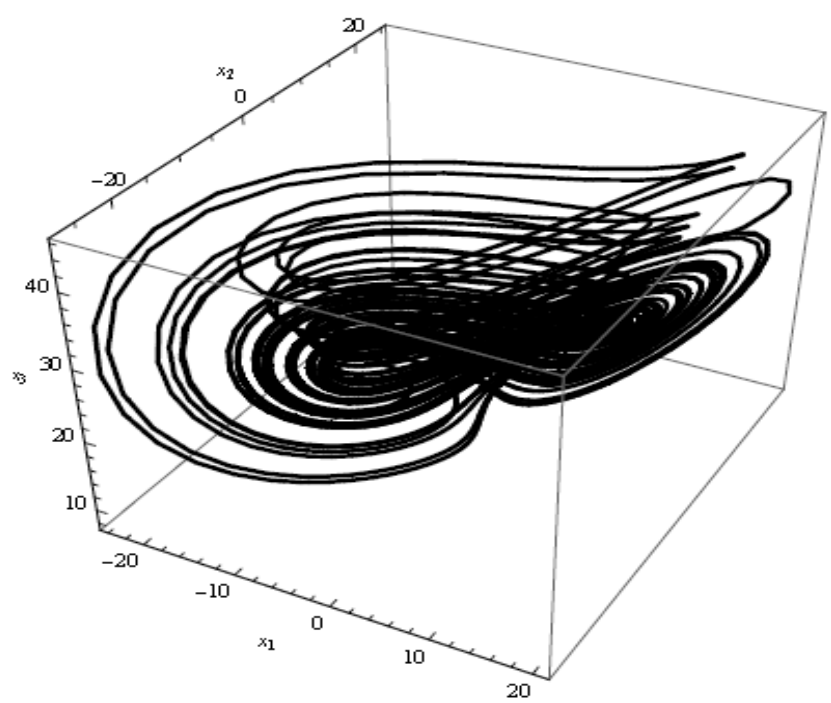

Figure 2. Chaotic attractor of Chen system when $\alpha=35, \beta=3$ and $\gamma=28$ 


\section{Adaptive synchronization of identical Pan systems}

In this study, we use adaptive control method to derive the synchronization of identical uncertain chaotic Pan System. Thus, the master system is described as follows.

$\dot{x}_{1}=a\left(x_{2}-x_{1}\right)$
$\dot{x}_{2}=c x_{1}-x_{1} x_{3}$
$\dot{x}_{3}=x_{1} x_{2}-b x_{3}$

where $x_{1}-x_{3}$ are the states variables $a, b$ and $c$ are unknown parameters of the system. The slave system is described by the controlled chaotic Pan dynamics.

$\dot{y}_{1}=a\left(y_{2}-y_{1}\right)+u_{1}$

$\dot{y}_{2}=c y_{1}-y_{1} y_{3}+u_{2}$

$\dot{y}_{3}=y_{1} y_{2}-b y_{3}+u_{3}$

where $y_{1}-y_{3}$ are states variables and $u_{1}-u_{3}$ are nonlinear controller to be designed.

The synchronization error is defined as follows.

$e_{i}=y_{i}-x_{i}(i=1,2,3)$

Then we get error dynamics as

$\dot{e}_{1}=a\left(e_{2}-e_{1}\right)+u_{1}$

$\dot{e}_{2}=c e_{1}-y_{1} y_{3}+x_{1} x_{3}+u_{2}$

$\dot{e}_{3}=y_{1} y_{2}-b e_{3}-x_{1} x_{2}+u_{3}$

Let us now define the Adaptive functions $u_{1}(t)-u_{3}(t)$ as.

$u_{1}(t)=-\widehat{a}\left(e_{2}-e_{1}\right)-k_{1} e_{1}$

$u_{2}(t)=-\hat{c} e_{1}+y_{1} y_{3}-x_{1} x_{3}-k_{2} e_{2}$

$u_{3}(t)=\widehat{b} e_{3}-y_{1} y_{2}+x_{1} x_{2}-k_{3} e_{3}$

where $\widehat{a}, \widehat{b}$ and $\widehat{c}$ are the estimates of $a, b$ and $c$, respectively and $k_{i}(i=1,2,3)$ are positive constants.

From (14) and (15) we get,

$\dot{e}_{1}=(a-\widehat{a})\left(e_{2}-e_{1}\right)-k_{1} e_{1}$

$\dot{e}_{2}=(c-\widehat{c}) e_{1}-k_{2} e_{2}$

$\dot{e}_{3}=-(b-\hat{b}) e_{3}-k_{3} e_{3}$

Let us now define the parameter estimation error as, 
$e_{a}=a-\hat{a}$

$e_{b}=b-\hat{b}$

$e_{c}=c-\widehat{c}$

From (16) and (17),

$\dot{e}_{1}=e_{a}\left(e_{2}-e_{1}\right)-k_{1} e_{1}$

$\dot{e}_{2}=e_{c} e_{1}-k_{2} e_{2}$

$\dot{e}_{3}=-e_{b} e-k_{3} e_{3}$

For the derivation of the update law for adjusting the estimates of the parameters, the Lyapunov approach is used, consider the quadratic Lyapunov function as,

$V=\frac{1}{2}\left(e_{1}^{2}+e_{2}^{2}+e_{3}^{2}+e_{a}^{2}+e_{b}^{2}+e_{c}^{2}\right)$

Which is a positive definite function on $R^{6}$, we also have

$\dot{e}_{a}=-\dot{\widehat{a}}$

$\dot{e}_{b}=-\dot{\vec{b}}$

$\dot{e}_{c}=-\dot{\hat{C}}$

Differentiating (19) along the trajectories of (18) and using (20), we get

$\dot{V}=e_{1} e_{a}\left(e_{2}-e_{1}\right)-k_{1} e_{1}^{2}+e_{1} e_{2} e_{c}-k_{2} e_{2}^{2}-e_{3}^{2} e_{b}-k_{3} e_{3}^{2}-\dot{\hat{a}} e_{a}-\dot{\vec{b}} e_{b}-\dot{\bar{c}} e_{c}$

From (21), the estimated parameters are updated by the following law.

$\dot{\hat{a}}=e_{1}\left(e_{2}-e_{1}\right)+k_{4} e_{a}$

$\dot{\vec{b}}=-e_{3}^{2}+k_{5} e_{b}$

$\dot{\hat{C}}=e_{1} e_{2}+k_{6} e_{c}$

where $k_{4}, k_{5}$ and $k_{6}$ are positive constants. From (21) and (22), we get

$\dot{V}=-k_{1} e_{1}^{2}-k_{2} e_{2}^{2}-k_{3} e_{3}^{2}-k_{4} e_{a}^{2}-k_{5} e_{b}^{2}-k_{6} e_{c}^{2}$

which is a negative definite function on $R^{6}$. Thus by Lyapunov stability theory [25], it is immediate that the synchronization error $e_{i}(i=1,2,3)$ and the parameter estimation error $e_{a}, e_{b}$ and $e_{c}$ decay to zero with time.

Result 3.1 The identical chaotic Pan systems are synchronized by adaptive control law (15), where the update law for the parameter estimates is given by (22) and $k_{i}(i=1,2,3,4,5,6)$ are positive constants. 
Numerical Result: To solve the (11) and (12) with the adaptive nonlinear controller (15), by using mathematica. We take $k_{i}=2(i=1,2,3,4,5,6)$. The parameters of the chaotic Pan systems is chosen as $a=10, b=8 / 3, c=16$. The initial values of parameter estimates are taken as $\widehat{a}(0)=10, \widehat{b}(0)=24, \widehat{c}(0)=20$ and the initial values of master and slave systems are chosen as $x_{1}(0)=15, x_{2}(0)=12, x_{3}(0)=32, y_{1}(0)=24, y_{2}(0)=20$ and $y_{3}(0)=16$, respectively. Figure 3 . Shows the synchronization of the Pan system and Figure 4. Shows the estimated values of the parameters, $\hat{a}, \hat{b}$ and $\hat{c}$ converge to the system parameters $a=10, b=8 / 3$ and $c=16$, respectively.
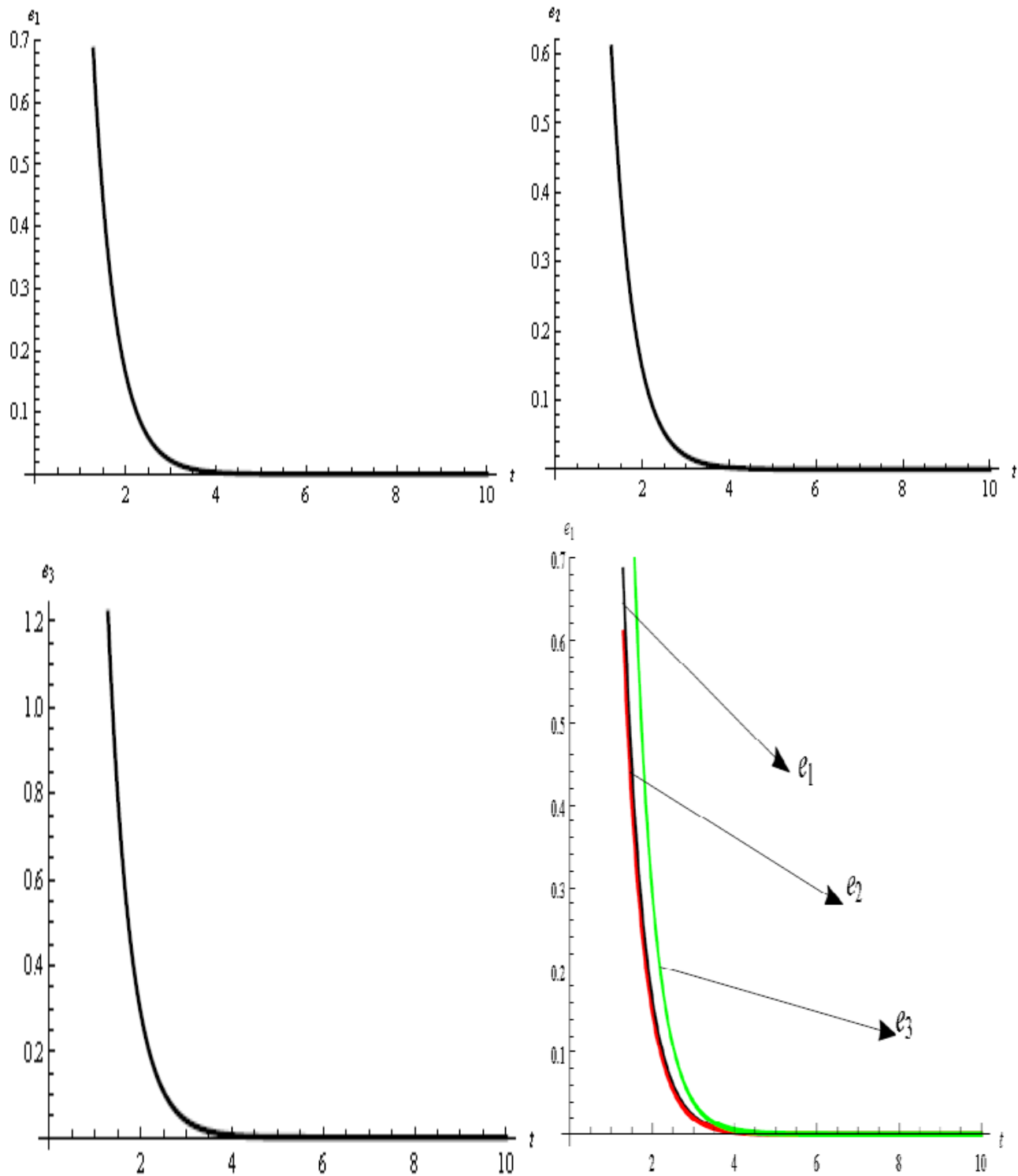

Figure 3. Synchronization of Pan system 


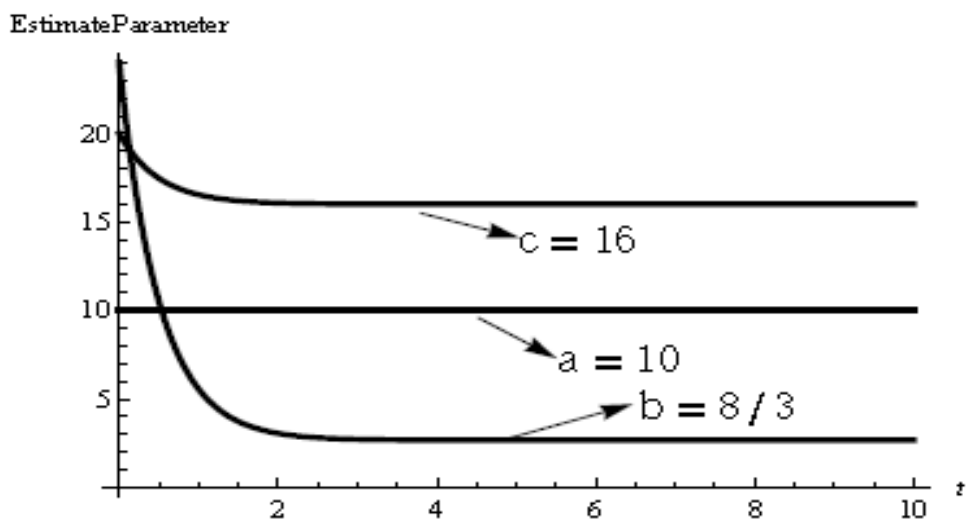

Figure 4. Adaptive parameters estimation errors: $\widehat{a}, \widehat{b}, \widehat{c}$

\section{Adaptive synchronization of two identical chaotic Chen systems}

The master system is given by

$\dot{x}_{1}=\alpha\left(x_{2}-x_{1}\right)$

$\dot{x}_{2}=\gamma x_{1}-\alpha x_{1}-x_{1} x_{3}+\gamma x_{2}$

$\dot{x}_{3}=x_{1} x_{2}-\beta x_{3}$

And slave system is

$\dot{y}_{1}=\alpha\left(y_{2}-y_{1}\right)+u_{1}$

$\dot{y}_{2}=\gamma y_{1}-\alpha y_{1}-y_{1} y_{3}+\gamma y_{2}+u_{2}$

$\dot{y}_{3}=y_{1} y_{2}-\beta y_{3}+u_{3}$

where $u_{1}-u_{3}$ are nonlinear controller to be designed.

The synchronization error is

$e_{i}=y_{i}-x_{i}(i=1,2,3)$

Then the error dynamics is defined as follows

$$
\begin{aligned}
& \dot{e}_{1}=\alpha\left(e_{2}-e_{1}\right)+u_{1} \\
& \dot{e}_{2}=\gamma\left(e_{1}+e_{2}\right)-\alpha e_{1}-y_{1} y_{3}+x_{1} x_{3}+u_{2} \\
& \dot{e}_{3}=y_{1} y_{2}-\beta e_{3}-x_{1} x_{2}+u_{3}
\end{aligned}
$$

Now defining the adaptive control functions $u_{1}(t)-u_{3}(t)$.

$$
\begin{aligned}
& u_{1}(t)=-\hat{\alpha}\left(e_{2}-e_{1}\right)-k_{1} e_{1} \\
& u_{2}(t)=-\hat{\gamma}\left(e_{1}+e_{2}\right)+\hat{\alpha} e_{1}+y_{1} y_{3}-x_{1} x_{3}-k_{2} e_{2} \\
& u_{3}(t)=-y_{1} y_{2}+\widehat{\beta} e_{3}+x_{1} x_{2}-k_{3} e_{3}
\end{aligned}
$$


where $\hat{\alpha}-\hat{\gamma}$ are estimates of $\alpha-\gamma$, respectively. and $k_{i}(i=1,2,3)$ are positive constants. Then the error dynamics becomes

$$
\begin{aligned}
& \dot{e}_{1}=(\alpha-\widehat{\alpha})\left(e_{2}-e_{1}\right)-k_{1} e_{1} \\
& \dot{e}_{2}=(\gamma-\widehat{\gamma})\left(e_{1}+e_{2}\right)-(\alpha-\hat{\alpha}) e_{1}-k_{2} e_{2} \\
& \dot{e}_{3}=-(\beta-\widehat{\beta}) e_{3}-k_{3} e_{3}
\end{aligned}
$$

The parameter error is given by

$$
\begin{aligned}
& e_{\alpha}=\alpha-\hat{\alpha} \\
& e_{\beta}=\beta-\hat{\beta} \\
& e_{\gamma}=\gamma-\hat{\gamma}
\end{aligned}
$$

From (29) and (30)

$$
\begin{aligned}
& \dot{e}_{1}=e_{\alpha}\left(e_{2}-e_{1}\right)-k_{1} e_{1} \\
& \dot{e}_{2}=e_{\gamma}\left(e_{1}+e_{2}\right)-e_{\alpha} e_{1}-k_{2} e_{2} \\
& \dot{e}_{3}=-e_{\beta} e_{3}-k_{3} e_{3}
\end{aligned}
$$

We consider the quadratic Lyapunov function is given by

$V=\frac{1}{2}\left(e_{1}^{2}+e_{2}^{2}+e_{3}^{2}+e_{\alpha}^{2}+e_{\beta}^{2}+e_{\gamma}^{2}\right)$

which is a positive definite function on $R^{6}$, we also noted that

$$
\begin{aligned}
& \dot{e}_{\alpha}=-\dot{\hat{\alpha}} \\
& \dot{e}_{\beta}=-\dot{\hat{\beta}} \\
& \dot{e}_{\gamma}=-\dot{\hat{\gamma}}
\end{aligned}
$$

Differentiating (32) we get

$$
\begin{aligned}
& \dot{V}=e_{1} e_{\alpha}\left(e_{2}-e_{1}\right)-k_{1} e_{1}^{2}+e_{\gamma} e_{2}\left(e_{1}+e_{2}\right)-e_{\alpha} e_{2} e_{1} \\
& -k_{2} e_{2}^{2}-e_{\beta} e_{3}^{2}-k_{3} e_{3}^{2}+e_{\alpha}\left[-e_{1}\left(e_{2}-e_{1}\right)+e_{1} e_{2}-k_{4} e_{\alpha}\right]+e_{\beta}\left(e_{3}^{2}-k_{5} e_{\beta}\right) \\
& +e_{\gamma}\left[-e_{2}\left(e_{1}+e_{2}\right)-k_{6} e_{\gamma}\right]
\end{aligned}
$$

The parameter update law is given by

$$
\begin{aligned}
& \dot{\hat{\alpha}}=e_{1}\left(e_{2}-e_{1}\right)-e_{1} e_{2}+k_{4} e_{\alpha} \\
& \dot{\hat{\beta}}=-e_{3}^{2}+k_{5} e_{\beta} \\
& \dot{\hat{\gamma}}=e_{2}\left(e_{1}+e_{2}\right)+k_{6} e_{\gamma}
\end{aligned}
$$

where $k_{4}, k_{5}, k_{6}$ are positive constants. 
Then (34) becomes

$\dot{V}=-k_{1} e_{1}^{2}-k_{2} e_{2}^{2}-k_{3} e_{3}^{2}-k_{4} e_{\alpha}^{2}-k_{5} e_{\beta}^{2}-k_{6} e_{\gamma}^{2}$

which is a negative definite function on $R^{6}$. Thus by Lyapunov stability theory [25], it is immediate that the synchronization error $e_{i}(i=1,2,3)$ and the parameter estimation error $e_{\alpha}, e_{\beta}$ and $e_{\gamma}$ decay to zero with time.

Result 4.1 The identical chaotic Chen systems are synchronized by adaptive control law (28), where the update law for the parameter estimates is given by (35) and $k_{i}(i=1,2,3,4,5,6)$ are positive constants.

Numerical Result: To solve the (24) and (25) with the adaptive nonlinear controller (28), by using the mathematica. We take $k_{i}=2(i=1,2,3,4,5,6)$. The parameters of the chaotic Chen systems is chosen as $\alpha=35, \beta=3, \gamma=28$. The initial values of parameter estimates are taken as $\hat{\alpha}(0)=17, \hat{\beta}(0)=4, \hat{\gamma}(0)=20$ and the initial values of master and slave systems are chosen as $x_{1}(0)=5, x_{2}(0)=18, x_{3}(0)=26, y_{1}(0)=20, y_{2}(0)=35$ and $y_{3}(0)=10$, respectively. Figure 5 . Shows the synchronization of the Chen system and Figure 6 . Shows the estimated values of the parameters, $\hat{\alpha}, \hat{\beta}$ and $\hat{\gamma}$ converge to the system parameters $\alpha=35, \beta=3$ and $\gamma=28$, respectively.

\section{Adaptive synchronization of two different Pan and Chen systems}

Here, we take Pan System as the master system and Chen system as the slave system is as follows.

$$
\begin{aligned}
& \dot{x}_{1}=a\left(x_{2}-x_{1}\right) \\
& \dot{x}_{2}=c x_{1}-x_{1} x_{3} \\
& \dot{x}_{3}=x_{1} x_{2}-b x_{3} \\
& \dot{y}_{1}=\alpha\left(y_{2}-y_{1}\right)+u_{1} \\
& \dot{y}=\gamma y_{1}-\alpha y_{1}-y_{1} y_{3}+\gamma y_{2}+u_{2} \\
& \dot{y}_{3}=y_{1} y_{2}-\beta y_{3}+u_{3}
\end{aligned}
$$

The synchronization error is defined as

$$
e_{i}=y_{i}-x_{i}(i=1,2,3)
$$

Then the error dynamics from (37) and (38) is as follows

$$
\begin{aligned}
& \dot{e}_{1}=\alpha\left(y_{2}-y_{1}\right)-a\left(x_{2}-x_{1}\right)+u_{1} \\
& \dot{e}_{2}=\gamma y_{1}-\alpha y_{1}-y_{1} y_{3}+\gamma y_{2}-c x_{1}+x_{1} x_{3}+u_{2} \\
& \dot{e}_{3}=y_{1} y_{2}-\beta y_{3}-x_{1} x_{2}+b x_{3}+u_{3}
\end{aligned}
$$

Defining the adaptive control function as 
$u_{1}(t)=-\hat{\alpha}\left(y_{2}-y_{1}\right)+\hat{a}\left(x_{2}-x_{1}\right)-k_{1} e_{1}$

$u_{2}(t)=-\hat{\gamma} y_{1}+\hat{\alpha} y_{1}+y_{1} y_{3}-\hat{\gamma} y_{2}+\widehat{c} x_{1}-x_{1} x_{3}-k_{2} e_{2}$

$u_{3}(t)=-y_{1} y_{2}-\widehat{\beta} y_{3}-x_{1} x_{2}+\widehat{b} x_{3}-k_{3} e_{3}$

Then the error dynamics becomes

$$
\begin{aligned}
& \dot{e}_{1}=(\alpha-\widehat{\alpha})\left(y_{2}-y_{1}\right)-(a-\widehat{a})\left(x_{2}-x_{1}\right)-k_{1} e_{1} \\
& \dot{e}_{2}=(\gamma-\widehat{\gamma}) y_{1}-(\alpha-\hat{\alpha}) y_{1}+(\gamma-\hat{\gamma}) y_{2}-(c-\widehat{c}) x_{1}-k_{2} e_{2} \\
& \dot{e}_{3}=-(\beta-\widehat{\beta}) y_{3}+(b-\widehat{b}) x_{3}-k_{3} e_{3}
\end{aligned}
$$
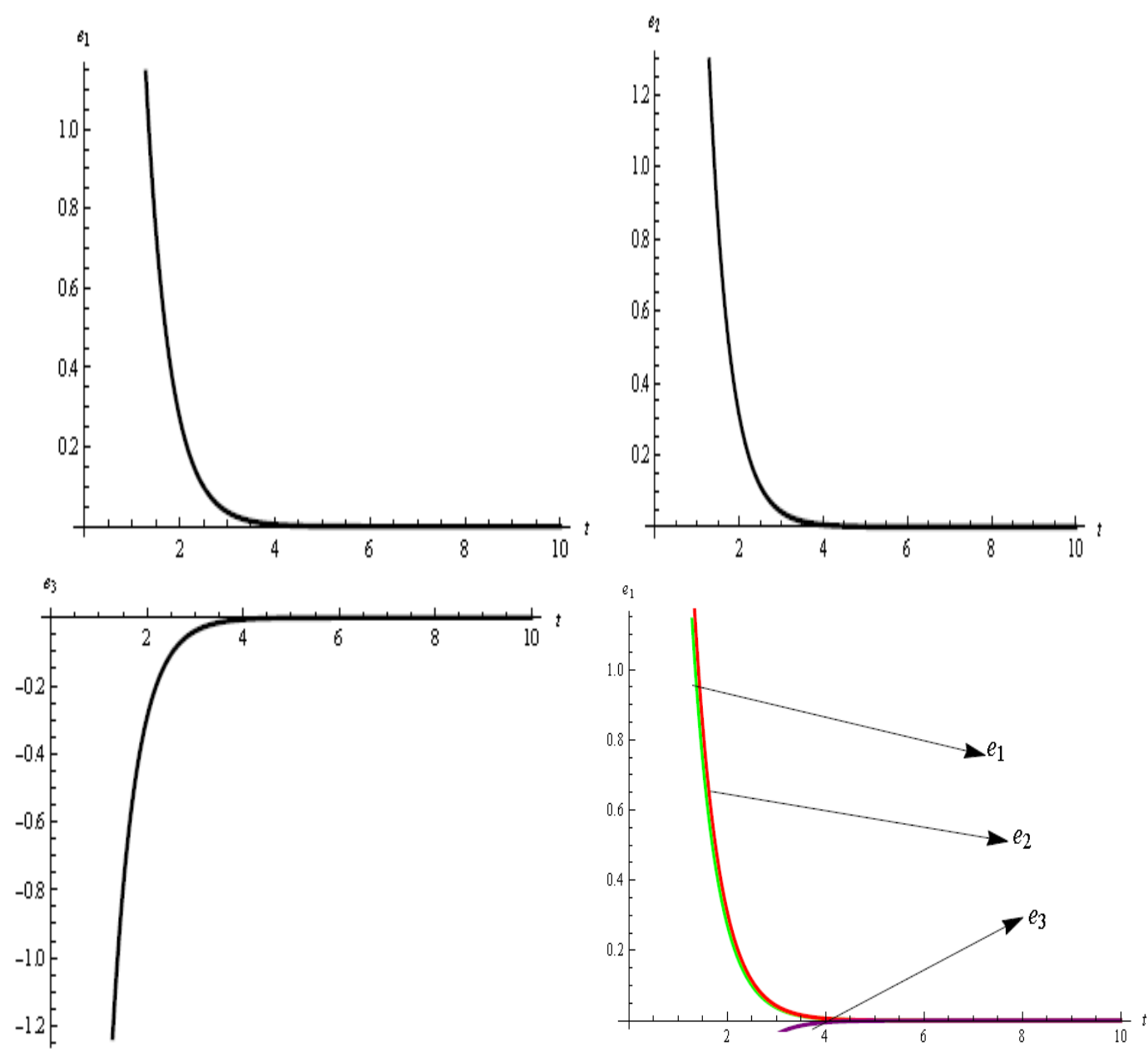

Figure 5. Synchronization of Chen system 


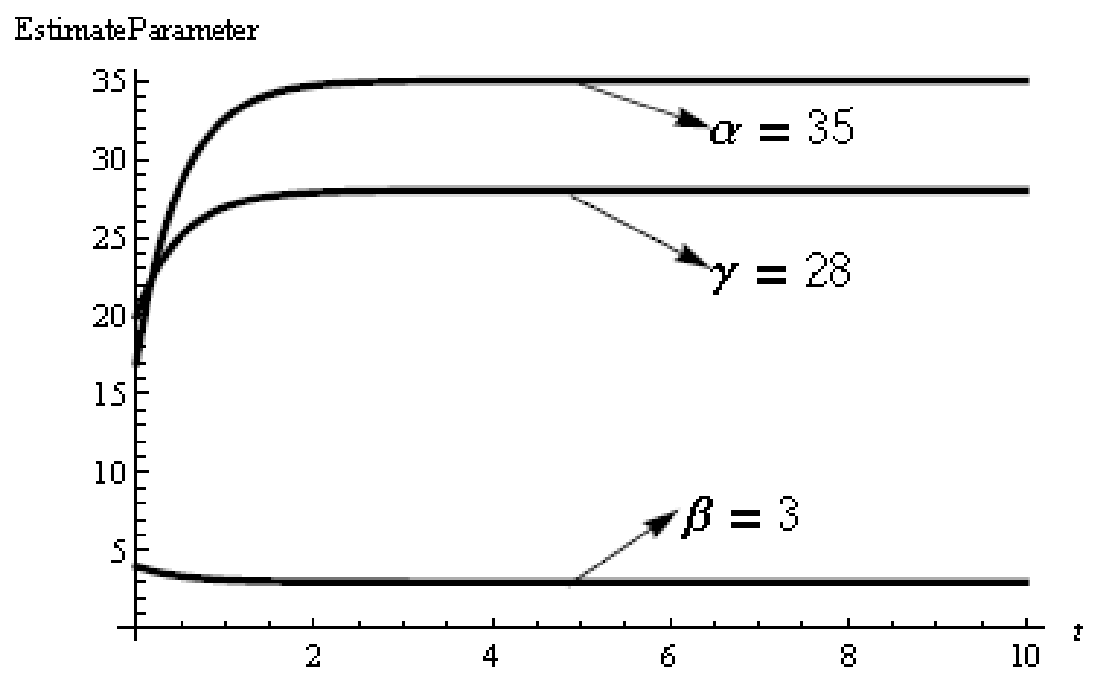

Figure 6. Adaptive parameters estimation errors: $\widehat{\alpha}, \widehat{\beta}, \hat{\gamma}$

The parameter estimation error is defined as

$$
\begin{aligned}
& e_{a}=a-\hat{a} \\
& e_{b}=b-\hat{b} \\
& e_{c}=c-\widehat{c} \\
& e_{\alpha}=\alpha-\hat{\alpha} \\
& e_{\beta}=\beta-\widehat{\beta} \\
& e_{\gamma}=\gamma-\hat{\gamma}
\end{aligned}
$$

From (42) and (43)

$$
\begin{aligned}
& \dot{e}_{1}=e_{\alpha}\left(y_{2}-y_{1}\right)-e_{a}\left(x_{2}-x_{1}\right)-k_{1} e_{1} \\
& \dot{e}_{2}=e_{\gamma} y_{1}-e_{\alpha} y_{1}+e_{\gamma} y_{2}-e_{c} x_{1}-k_{2} e_{2} \\
& \dot{e}_{3}=-e_{\beta} y_{3}+e_{b} x_{3}-k_{3} e_{3}
\end{aligned}
$$

Chose the quadratic Lyapunov function as

$V=\frac{1}{2}\left(e_{1}^{2}+e_{2}^{2}+e_{3}^{2}+e_{a}^{2}+e_{b}^{2}+e_{c}^{2}+e_{\alpha}{ }^{2}+e_{\beta}{ }^{2}+e_{\gamma}{ }^{2}\right)$

which is positive definite function on $R^{9}$, we also have

$$
\begin{aligned}
& \dot{e}_{\alpha}=-\dot{\hat{\alpha}}, \dot{e}_{\beta}=-\dot{\hat{\beta}} \\
& \dot{e}_{\gamma}=-\dot{\hat{\gamma}}, \dot{e}_{a}=-\dot{\hat{a}} \\
& \dot{e}_{b}=-\dot{\hat{b}}, \quad \dot{e}_{b}=-\dot{\hat{C}}
\end{aligned}
$$


The parameters estimated update law is defined as

$\dot{\hat{\alpha}}=e_{1}\left(y_{2}-y_{1}\right)-e_{2} y_{1}+k_{4} e_{\alpha}$

$\dot{\hat{\beta}}=-e_{3} y_{3}+k_{5} e_{\beta}$

$\dot{\hat{\gamma}}=y_{1} e_{2}+y_{2} e_{2}+k_{6} e_{\gamma}$

$\dot{\hat{a}}=-e_{1}\left(x_{2}-x_{1}\right)+k_{7} e_{a}$

$\dot{\vec{b}}=x_{3} e_{3}+k_{8} e_{b}$

$\dot{\hat{c}}=-e_{2} x_{1}+k_{9} e_{c}$

Now differentiating (45) we get

$\dot{V}=-k_{1} e_{1}{ }^{2}-k_{2} e_{2}{ }^{2}-k_{3} e_{3}{ }^{2}-k_{4} e_{\alpha}{ }^{2}-k_{5} e_{\beta}{ }^{2}-k_{6} e_{\gamma}{ }^{2}-k_{7} e_{a}{ }^{2}-k_{8} e_{b}{ }^{2}-k_{9} e_{c}{ }^{2}$

which is a negative definite function on $R^{9}$. Thus by Lyapunov stability theory [25], it is immediate that the synchronization error $e_{i} \quad(i=1,2,3)$ and the parameter estimation error $e_{\alpha}, e_{\beta}, e_{\gamma}, e_{a}, e_{b}$ and $e_{c}$ decay to zero with time.

Result 5.1 The chaotic Pan and Chen systems are synchronized by adaptive control law (41), where the update law for the parameter estimates is given by $(47)$ and $k_{i}(i=1,2,3,4,5,6)$ are positive constants.

Numerical Result: To solve the (37) and (38) with the adaptive nonlinear controller (41), by using the mathematica. We take $k_{i}=2(i=1,2,3,4,5,6)$. The parameters of the chaotic Pan and Chen systems are chosen as $a=10, b=8 / 3, c=16$ and $\alpha=35, \beta=3, \gamma=28$, respectively. The initial values of parameter estimates are taken as $\hat{\alpha}(0)=17, \widehat{\beta}(0)=4, \widehat{\gamma}(0)=20$, $\widehat{a}(0)=10, \widehat{b}(0)=24, \widehat{c}(0)=20$ and the initial values of master and slave systems are chosen as $x_{1}(0)=15, x_{2}(0)=12, x_{3}(0)=32, y_{1}(0)=5, y_{2}(0)=18$ and $y_{3}(0)=26$, respectively. Figure 7 . Shows the synchronization of the Pan and Chen system and Figure: 8. Shows the estimated values of the parameters $\hat{\alpha}, \hat{\beta}, \hat{\gamma}, \widehat{a}, \hat{b}$ and $\widehat{c}$ converge to the system parameters $\alpha=35, \beta=3$, $\gamma=28, a=10, b=8 / 3$ and $c=16$ respectively.

\section{Conclusion}

In this paper, we apply adaptive nonlinear control method for chaos synchronization of identical Pan Systems, identical Chen systems and nonidentical Pan and Chen systems with unknown parameters. The adaptive synchronization results derived in this paper are established using Lyapunov stability theory. Numerical results are shown the effectiveness of the synchronization scheme. 

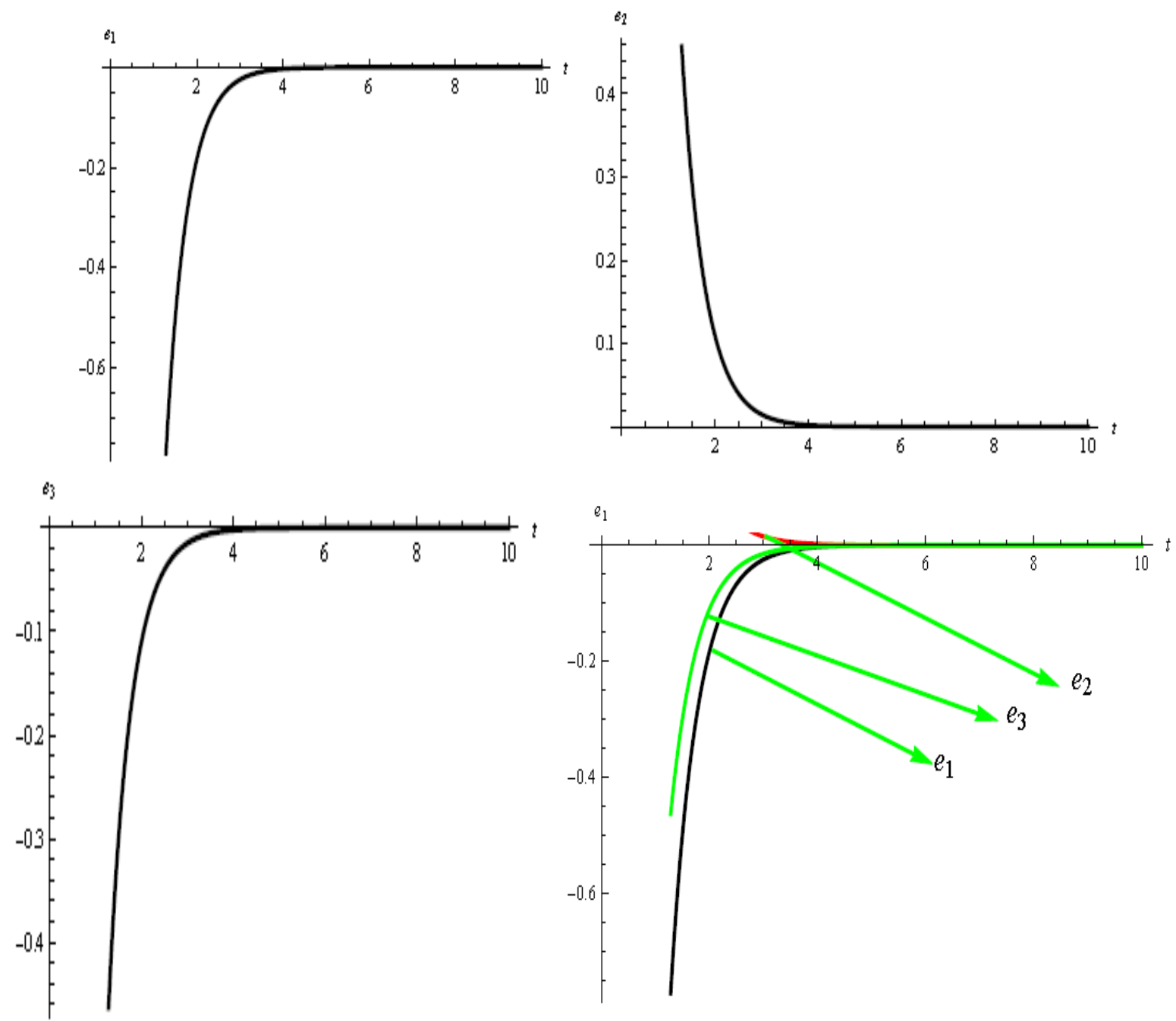

Figure 7. Synchronization of Pan and Chen systems

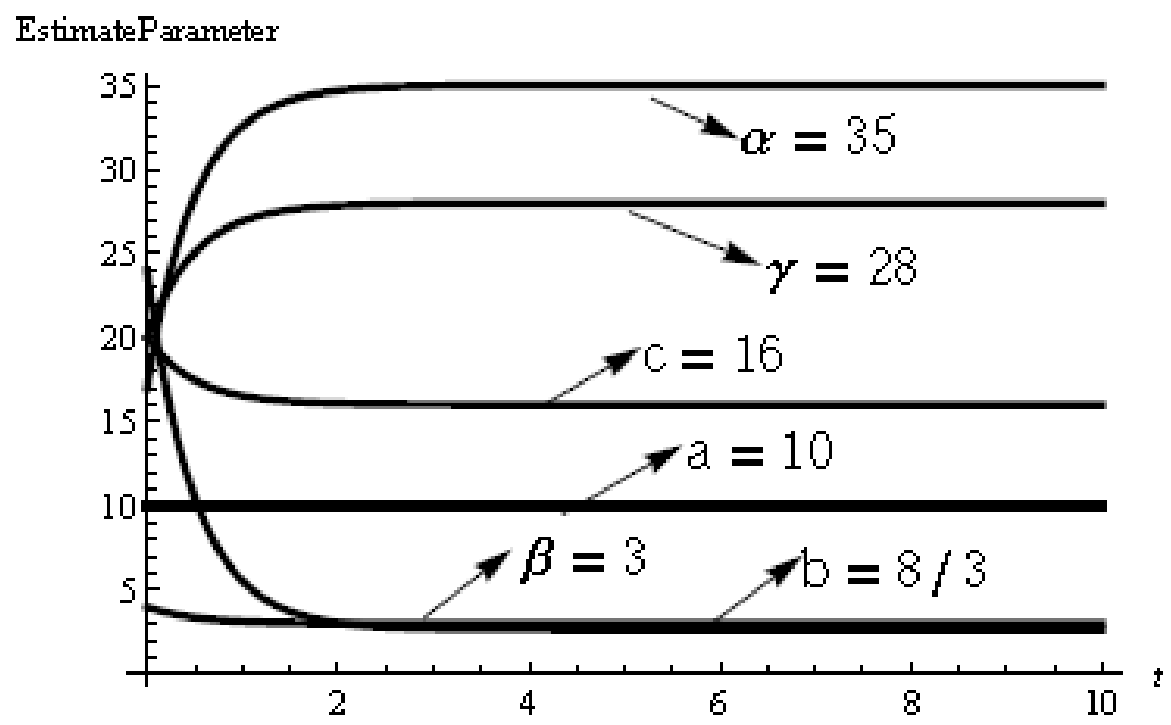

Figure 8. Adaptive parameters estimation errors: $\widehat{a}, \widehat{b}, \widehat{c}, \hat{\alpha}, \widehat{\beta}, \widehat{\gamma}$ 


\section{References}

[1] Lorenz, E. N., “Deterministic non-periodic flows”, J. Atoms. Sci. 20 (1963) : 130-141.

[2] Ott, E., Grebogi, C., Yorke, J.A., “Controlling chaos”, Phys.Rev.Lett. 62(2) (1990) : 821824.

[3] Pecora, L.M., Carroll, T.L., “Synchronization in chaotic system”, Phys, Rev. Lett. 64(8) (1990) : 821-824.

[4] Cuomo, K.M., Oppenheim, A.V., Strogatz, S.H., "Synchronization of Lorenze-based chaotic circuits with applications to communication”, IEEE Trans. Circuits syst. II, Express Briefs 40(10) (1993) : 626-633.

[5] Ying, T., Chua, L.O., "Impulsive control and synchronization of nonlinear dynamical systems and application to secure communication”, Int. J. Bifurc. Chaos 7(3) (1997) : 645-665.

[6] Zhang, H.G., Ma, T.D., Huang, G.B., Wang, Z.L., "Robust global exponential synchronization of uncertain chaotic delayed neural networks via dual stage impulsive control”, IEEE Trans, Syst. Man Cybern., Part B, Cybern. 40(3), (2010) : 831-844.

[7] Guan, X.P., Fan, Z.P., Chen,C.L., "Chaos control and its application in secure communication”, National Defence Industry Press Beijing (2002).

[8] Pan, L., Zhou, W.N., Fang, A., Li, D.Q., "A novel active pinning control for synchronization and anti-synchronization of new uncertain unified chaotic systems", Nonlinear Dyn.62(1-2) (2010) : 417-425.

[9] Sundar, S., Minai, A.A., "Synchronization of randomly multiplexed chaotic systems with applications to communication”, Phys. Rev. Lett. 85(25) (2000) : 5456-5459.

[10] Feki, M., “An adaptive chaos synchronization scheme applied to secure communication”, Chaos Solitons Fractals 18(1) (2003) : 141-148

[11] Yang, S.S., Duan, C.K., "Generalized synchronization in chaotic systems”, Chaos Solitons Fractals 9(10) (1998) : 1703-1707.

[12] Wang, Y.W., Guan, Z.H., “Generalized synchronization of continuous chaotic system”, Chaos Solitons Fractals 27(1) (2006) : 97-101.

[13] Michael, G.R., Arkady, S.P., Jurgen, K., "Phase synchronization of chaotic oscillators”, Phys. Rev. Lett. 76(11) (1996) : 1804-1807.

[14] Ho, M.C., Hung, Y.C., Chou, C.H., "Phase and anti-phase synchronization of two chaotic systems by using active control”. Phys. Lett. A 296(1) (2002) : 43-48.

[15] Di, L.C., Liao, X.F., Wong, D.W., "Lag synchronization of hyperchaos with application to secure communications”, Chaos Solitons Fractals 23(1) (2005) : 183-193.

[16] Zhang, Y., Sun, J., "Chaotic synchronization and anti -synchronization based on suitable separation”, Phys. Lett. A 330(6) (2004) : 442-447.

[17] Ricardo, A.L., Rafael, M.G., "Synchronization of a class of chaotic signals via robust observer design”, Chaos Solitons Fractals 37(2) (2008) : 581-587.

[18] Salarieh, H., Alasty, A., "Adaptive synchronization of two chaotic systems with stochastic unknown parameters”, Commun Nonlinear Sci. Numer. Simul. 14(2) (2009) : 508-519.

[19] Chen, G.H., "Controlling chaos and chaotification in the Chen-Lee system by multiple time delays”, Chaos Solitons Fractals 36(4) (2008) : 843-852. 
[20] Yau, H.T., Shieh, C.S., “Chaos synchronization using fuzzy logic controller”, Nonlinear Anal., Real World Appl. 9(4) (2008) : 1800-1810.

[21] Hu, M.F., Xu, Z.Y., “Adaptive feedback controller for projective synchronization”, Nonlinear Anal., Real World Appl. 9(3) (2008) : 1253-1260.

[22] Ghosh, D., Bhattacharya, S., "Projective synchronization of new hyperchaotic system with fully unknown parameters”, Nonlinear Dyn. 61(1-2) (2010) : 11-21.

[23] Liu, Y.J., Tong, S.C., Wang, W., Li, Y.M., “Observer-based direct adaptive fuzzy control of uncertain nonlinear systems and its applications”, Int. J. Control. Autom. Syst. 7(4) (2009) : 681-690.

[24] Elabbasy, E.M., Agiza, H.N., Dessoky, M.M., “Adaptive synchronization of a hyperchaotic system with uncertain parameter”, Chaos Solitons Fractals 30(5) (2006) : 11-33.

[25] Hahn, W., “The stability of motion”, Springer”, New York (1967).

[26] Khan, A., Shikha, N.A., "Robust adaptive sliding mode control technique for combination synchronisation of non-identical time delay chaotic systems”, International Journal of Modelling, Identification and Control 31.3 (2019) : 268-277.

[27] Khan, A., Shikha, S., Azar, A.T., "Combination-Combination AntiSynchronization of Four Fractional Order Identical Hyperchaotic Systems", International Conference on Advanced Machine Learning Technologies and Applications Springer, Cham (2019).

[28] Bhat, M.A., Shikha, N.A., "Complete synchronisation of non-identical fractional order hyperchaotic systems using active control", International Journal of Automation and Control 13.2 (2019) : 140-157.

[29] Shikha, S., Azar, A.T., Zhu, Q., "Multi-switching Master-Slave Synchronization of Nonidentical Chaotic Systems", Innovative Techniques and Applications of Modelling, Identification and Control. Springer Singapore (2018) : 321-330.

[30] Vaidyanathan, S., Azar, A. T., Sambas, A., Shikha, S., Alain, K. S. T., Serrano, F. E., “A novel hyperchaotic system with adaptive control, synchronization, and circuit simulation In Advances in System Dynamics and Control”, IGI Global (2018) : 382-419. 